\title{
REMARK ON THE WELL-POSEDNESS OF WEAKLY DISPERSIVE EQUATIONS
}

\author{
JiaO HE ${ }^{1}$ AND YOUCEF MAMmeri ${ }^{2,3}$
}

\begin{abstract}
We improve the results about the well-posedness of the regularized fractional dispersive equation $\left(1+D_{x}^{\alpha}\right) u_{t}+u_{x}+u u_{x}=0$ when $0<\alpha \leq 1$. When $\alpha<1$, the existence and uniqueness of vanishing viscosity solution is proved.
\end{abstract}

\section{INTRODUCTION}

Fractional Benjamin-Bona-Mahony (fBBM) equation

$$
u_{t}+u_{x}+u u_{x}+D_{x}^{\alpha} u_{t}=0
$$

was introduced by Linares, Pilod and Saut [16] to investigate the role of weak dispersion $(0<\alpha<1)$ on the solution of the Burgers equation

$$
u_{t}+u_{x}+u u_{x}=0 .
$$

They showed the local in time well-posedness in Sobolev spaces using energy estimates. Nevertheless, this method does not provide the uniqueness. Indeed, the difference $w=u-v$ between two solutions satisfies (see inequality (4.31) of [16])

$$
\begin{aligned}
\frac{d}{d t}\|w\|_{H^{s+\frac{\alpha}{2}}}^{2} \leq & \int_{\mathbb{R}}\left|\left((-\Delta)^{s / 2}(u+v)\right) w_{x}(-\Delta)^{s / 2} w\right| d x+\frac{1}{2} \int_{\mathbb{R}}\left|(u+v)_{x}(-\Delta)^{s / 2} w\right|^{2} d x \\
& +\int_{\mathbb{R}}\left|R(-\Delta)^{s / 2} w\right| d x+\int_{\mathbb{R}}\left|\left((-\Delta)^{s / 2}(u+v)_{x}\right) w(-\Delta)^{s / 2} w\right| d x,
\end{aligned}
$$

where $\int_{\mathbb{R}}\left|R(-\Delta)^{s / 2} w\right| d x \leq\|u+v\|_{H^{s+\frac{\alpha}{2}}}\|w\|_{H^{s+\frac{\alpha}{2}}}^{2}$. But the last term can not be uniformly controlled by the $H^{s+\frac{\alpha}{2}}-$ norm if $0<\alpha<1$.

When $0<\alpha<1$, it seems difficult to obtain the global well-posedness. It has been shown by Bona and Saut [3] that the linearization around 0 has blow-up solution and Klein, Saut numerically observe blow-up when $0<\alpha<\frac{1}{3}$ [15]. While $\alpha=1$, global well-posedness can be obtained thanks to Brezis-Gallouët estimates [5,18].

The paper is organized as follows. In Section 2, we improve the regularity of the global well-posedness when $\alpha=1$. In Section 3, we deal with the uniqueness of vanishing viscosity solution for $0<\alpha<1$.

\footnotetext{
1 Institut Camille Jordan, CNRS UMR 5208, Université Claude Bernard Lyon 1, 69622 Villeurbanne, France; e-mail: jiao.he@math.univ-lyon1.fr

2 Laboratoire Amiénois de Mathématique Fondamentale et Appliquée, CNRS UMR 7352, Université de Picardie Jules Verne, 80069 Amiens, France

3 Institut de Génétique, Environnement et Protection des Plantes, INRA UMR 1349, 35650 Le Rheu, France;

e-mail: youcef .mammeri@u-picardie.fr

(C) EDP Sciences, SMAI 2018
} 


\section{The Regularized Benjamin-Ono equation}

When $\alpha=1$, the equation can be rewritten as the Benjamin-Ono equation under the form

$$
u_{t}+H\left(u_{x}\right)_{t}+u_{x}+u u_{x}=0
$$

where $H$ is the Hilbert defined by its Fourier symbol $\widehat{H(u)}(\xi)=-i \operatorname{sgn}(\xi) \hat{u}(\xi)$.

Theorem 1.1. Let $\alpha=1$ and $u_{0} \in H^{\frac{1}{2}}(\mathbb{R})$. There exists a unique solution $u \in \mathcal{C}\left(\mathbb{R} ; H^{\frac{1}{2}}(\mathbb{R})\right)$ of the initial value problem

$$
\left\{\begin{array}{l}
u_{t}+u_{x}+u u_{x}+D_{x} u_{t}=0 \\
u(x, 0)=u_{0}(x)
\end{array}\right.
$$

Moreover, for all $t \in \mathbb{R}$

$$
\|u(t)\|_{H^{\frac{1}{2}}}=\left\|u_{0}\right\|_{H^{\frac{1}{2}}}
$$

and the map $u_{0} \in H^{\frac{1}{2}}(\mathbb{R}) \rightarrow u \in \mathcal{C}\left(\mathbb{R} ; H^{\frac{1}{2}}(\mathbb{R})\right)$ is continuous.

The proof is done in two steps: first, a compactness argument is used to obtain a weak solution, then the uniqueness of the weak solution provides the strong continuity of the weak solution.

Let $\left(u_{n, 0}\right)_{n \in \mathbb{N}}$ be a sequence of $H^{1}(\mathbb{R})$ such that $u_{n, 0} \rightarrow u_{0}$ in $H^{\frac{1}{2}}(\mathbb{R})$. We denote by $u_{n}(t) \in \mathcal{C}\left(\mathbb{R} ; H^{1}(\mathbb{R})\right)$ the solution of the initial value problem associated with the initial datum $u_{n, 0}$. Then it is proved that the energy $[18]$

is preserved with respect to time, i.e.

$$
E(t)=\int_{\mathbb{R}} u_{n}^{2}+\left.\left.|| D_{x}\right|^{\frac{1}{2}} u_{n}\right|^{2} d x
$$

$$
\left\|u_{n}\right\|_{H^{\frac{1}{2}}}^{2}=\left\|u_{n, 0}\right\|_{H^{\frac{1}{2}}}^{2} \leq C
$$

and the sequence $\left(u_{n}\right)_{n \in \mathbb{N}}$ is bounded in $\mathcal{C}\left(\mathbb{R} ; H^{\frac{1}{2}}(\mathbb{R})\right)$.

On the other hand, multiplying (1) by $\partial_{t} u_{n}$ and integrating over space gives

$$
\int_{\mathbb{R}}\left|\partial_{t} u_{n}\right|^{2} d x=-\int_{\mathbb{R}} \partial_{t} u_{n} \partial_{x}\left(1+D_{x}\right)^{-1}\left(u_{n}+\frac{u_{n}^{2}}{2}\right) d x,
$$

and Young's inequality provides, for $\varepsilon>0$.

$$
\begin{aligned}
\left\|\partial_{t} u_{n}\right\|_{L^{2}}^{2}=\int_{\mathbb{R}}\left|\partial_{t} u_{n}\right|^{2} d x & \leq\left|\int_{\mathbb{R}} \partial_{t} u_{n} \partial_{x}\left(1+D_{x}\right)^{-1} u_{n} d x+\int_{\mathbb{R}} \partial_{t} u_{n} \partial_{x}\left(1+D_{x}\right)^{-1} \frac{u_{n}^{2}}{2} d x\right| \\
& \leq \varepsilon \int_{\mathbb{R}}\left|\partial_{t} u_{n}\right|^{2} d x+\frac{1}{2 \varepsilon} \int_{\mathbb{R}}\left|\partial_{x}\left(1+D_{x}\right)^{-1} u_{n}\right|^{2} d x+\frac{1}{2 \varepsilon} \int_{\mathbb{R}}\left|\partial_{x}\left(1+D_{x}\right)^{-1} \frac{u_{n}^{2}}{2}\right|^{2} d x \\
& \leq \varepsilon\left\|\partial_{t} u_{n}\right\|_{L^{2}}^{2}+\frac{1}{2 \varepsilon}\left\|\partial_{x}\left(1+D_{x}\right)^{-1} u_{n}\right\|_{L^{2}}^{2}+\frac{1}{2 \varepsilon}\left\|\partial_{x}\left(1+D_{x}\right)^{-1} \frac{u_{n}^{2}}{2}\right\|_{L^{2}}^{2} \\
& \leq \varepsilon\left\|\partial_{t} u_{n}\right\|_{L^{2}}^{2}+\frac{1}{4 \varepsilon}\left(\left\|u_{n}\right\|_{L^{2}}^{2}+\left\|u_{n}^{2}\right\|_{L^{2}}^{2}\right) \\
& \leq \varepsilon\left\|\partial_{t} u_{n}\right\|_{L^{2}}^{2}+\frac{1}{4 \varepsilon}\left(\left\|u_{n}\right\|_{L^{2}}^{2}+\left\|u_{n}\right\|_{L^{4}}^{4}\right) \\
& \leq \varepsilon\left\|\partial_{t} u_{n}\right\|_{L^{2}}^{2}+\frac{1}{4 \varepsilon}\left(\left\|u_{n}\right\|_{L^{2}}^{2}+\left\|u_{n}\right\|_{H^{\frac{1}{2}}}^{4}\right) .
\end{aligned}
$$

Taking $\varepsilon=\frac{1}{2}$, it gets

$$
\left\|\partial_{t} u_{n}\right\|_{L^{2}} \leq\left\|u_{n}\right\|_{H^{\frac{1}{2}}}^{2} \leq\left\|u_{n, 0}\right\|_{H^{\frac{1}{2}}}^{2} \leq C .
$$


Thus,

$$
\left\|u_{n}(t)-u_{n}(s)\right\|_{L^{2}}=\left\|\int_{s}^{t} \partial_{t} u_{n}(r) d r\right\|_{L^{2}} \leq \int_{s}^{t}\left\|\partial_{t} u_{n}(r)\right\|_{L^{2}} d r \leq C|t-s|,
$$

and the sequence $\left(u_{n}\right)_{n \in \mathbb{N}}$ is uniformly bounded and equicontinuous.

We deduce according to the Rellich theorem for all $T>0$, there exists $u \in \mathcal{C}_{w}\left([0, T] ; H^{\frac{1}{2}}(\mathbb{R})\right) \bigcap \mathcal{C}\left([0, T] ; L^{2}(\mathbb{R})\right)$ and a subsequence $\left(u_{n_{k}}\right)_{k \in \mathbb{N}}$ such that $\forall t \in[0, T]$

$$
u_{n_{k}}(t) \rightarrow u(t) \text { in } H^{\frac{1}{2}}(\mathbb{R}) \text { and } u_{n_{k}}(t) \rightarrow u(t) \text { in } L^{2}(\mathbb{R})
$$

Since the subsequence $\left(u_{n_{k}}\right)_{k \in \mathbb{N}}$ satisfies for all $v \in \mathcal{C}_{c}^{\infty}(\mathbb{R})$

$$
\int_{0}^{T} \int_{\mathbb{R}} v \partial_{t} u_{n_{k}}+v \partial_{t} D_{x} u_{n_{k}}+v \partial_{x}\left(u_{n_{k}}+\frac{u_{n_{k}}^{2}}{2}\right) d x d t=0,
$$

the limit verifies

$$
\int_{0}^{T} \int_{\mathbb{R}} u \partial_{t} v+u \partial_{t} D_{x} v+\left(u+\frac{u^{2}}{2}\right) \partial_{x} v d x d t=0 .
$$

The function $u \in \mathcal{C}_{w}\left([0, T] ; H^{\frac{1}{2}}(\mathbb{R})\right) \bigcap \mathcal{C}\left([0, T] ; L^{2}(\mathbb{R})\right)$ is a weak solution of the equation $(1)$ and

$$
\|u(t)\|_{H^{\frac{1}{2}}} \leq \liminf _{k \rightarrow \infty}\left\|u_{n_{k}}(t)\right\|_{H^{\frac{1}{2}}} \leq\left\|u_{0}\right\|_{H^{\frac{1}{2}}} .
$$

Suppose now that the weak solution of the Cauchy problem $u \in \mathcal{C}_{w}\left([0, T] ; H^{\frac{1}{2}}(\mathbb{R})\right) \bigcap \mathcal{C}\left([0, T] ; L^{2}(\mathbb{R})\right)$ is unique. By reversing time in the regularized Benjamin-Ono equation (1), we have

$$
\|u(-t)\|_{H^{\frac{1}{2}}} \leq\|u(0)\|_{H^{\frac{1}{2}}} \text { or }\|u(0)\|_{H^{\frac{1}{2}}} \leq\|u(t)\|_{H^{\frac{1}{2}}}
$$

From inequality (2) and from the uniqueness of the weak solution, we obtain

$$
\|u(t)\|_{H^{\frac{1}{2}}}=\left\|u_{0}\right\|_{H^{\frac{1}{2}}}
$$

and the solution $u$ belongs to $\mathcal{C}\left([0, T] ; H^{\frac{1}{2}}(\mathbb{R})\right)$. Note that we also obtain the continuity with respect to the initial data since we proved that for $u_{n, 0} \rightarrow u_{0}$ in $H^{\frac{1}{2}}(\mathbb{R})$, then the respective solution $\left(u_{n}\right)$ verifies $u_{n} \rightarrow u$ in $\mathcal{C}\left([0, T] ; H^{\frac{1}{2}}(\mathbb{R})\right)$.

It remains to prove the uniqueness of the weak solution. We are inspired by the method introduced by Yudovich [21]. We need a Trudinger-type estimates proved by Gérard and Grellier [9] in the torus $\mathbb{T}$.

Lemma 1.2. There exists a constant $C>0$ such that for all $2<p<\infty$, we have

$$
\|u\|_{L^{p}(\mathbb{R})} \leq C \sqrt{p}\|u\|_{H^{\frac{1}{2}(\mathbb{R})}} .
$$

Proof. The proof is similar to [9] except that $u$ is split as

$$
u=u_{>\lambda}+u_{<\lambda}=\frac{1}{2 \pi} \int_{|\xi| \leq \lambda} e^{i \xi x} \widehat{u}(\xi) d \xi+\frac{1}{2 \pi} \int_{|\xi| \geq \lambda} e^{i \xi x} \widehat{u}(\xi) d \xi
$$


Let $u$ and $v \in \mathcal{C}_{w}\left([0, T] ; H^{\frac{1}{2}}(\mathbb{R})\right) \cap \mathcal{C}\left([0, T] ; L^{2}(\mathbb{R})\right)$ be two weak solutions of (1) starting from the same initial datum. Consider the function $g$ defined as

$$
g(t)=\|u(t)-v(t)\|_{L^{2}}^{2} .
$$

Then, for $w:=u-v$ and $P\left(D_{x}\right):=\partial_{x}\left(1+D_{x}\right)^{-1}$, we have

$$
\begin{aligned}
g^{\prime}(t) & =2 \int_{\mathbb{R}}(u-v)\left(u_{t}-v_{t}\right) d x=-2 \int_{\mathbb{R}}(u-v) P\left(D_{x}\right)\left(u+\frac{u^{2}}{2}-v-\frac{v^{2}}{2}\right) \\
& =-2 \int_{\mathbb{R}} w P\left(D_{x}\right)(w)+w P\left(D_{x}\right)(w(u+v)) d x=-2 \int_{\mathbb{R}} w P\left(D_{x}\right)(w(u+v)) d x \\
& =-\int_{\mathbb{R}} w^{2} P\left(D_{x}\right)(u+v) d x-\int_{\mathbb{R}} w\left(P\left(D_{x}\right) w\right)(u+v) d x-\int_{\mathbb{R}} w R d x \\
& =: \mathrm{I}+\mathrm{II}+\mathrm{III}
\end{aligned}
$$

where

$$
R=P\left(D_{x}\right)(w(u+v))-(u+v) P\left(D_{x}\right) w-w P\left(D_{x}\right)(u+v) .
$$

Since $w^{2}=w^{2\left(1-\frac{1}{p}\right)} w^{\frac{2}{p}}$, the Hölder inequality provides

$$
\begin{aligned}
|\mathrm{I}|=\int_{\mathbb{R}} w^{2} P\left(D_{x}\right)(u+v) d x & \leq\left\|w^{2\left(1-\frac{1}{p}\right)}\right\|_{L^{\frac{p}{p-1}}}\left\|w^{\frac{2}{p}} P\left(D_{x}\right)(u+v)\right\|_{L^{p}} \\
& \lesssim\|w\|_{L^{2}}^{2\left(1-\frac{1}{p}\right)}\left(\|u\|_{L^{4}}^{\frac{2}{p}}+\|v\|_{L^{4}}^{\frac{2}{p}}\right)\left\|P\left(D_{x}\right)(u+v)\right\|_{L^{2 p}} .
\end{aligned}
$$

We note that the operator $P\left(D_{x}\right)$ is bounded in $L^{p}$, for $1<p<+\infty$ according to the Mikhlin-Hörmander theorem $[17,19]$. The Sobolev and the Trudinger inequalities imply

$$
|\mathrm{I}| \lesssim\|w\|_{L^{2}}^{2\left(1-\frac{1}{p}\right)}\left(\|u\|_{L^{4}}^{\frac{2}{p}}+\|v\|_{L^{4}}^{\frac{2}{p}}\right)\left(\|u\|_{L^{2 p}}+\|v\|_{L^{2 p}}\right) \lesssim \sqrt{2 p} g(t)^{1-\frac{1}{p}}\left(\|u\|_{L^{4}}^{\frac{2}{p}}+\|v\|_{L^{4}}^{\frac{2}{p}}\right)\left(\|u\|_{H^{\frac{1}{2}}}+\|v\|_{H^{\frac{1}{2}}}\right)
$$

and from inequality $(2)$

$$
|\mathrm{I}| \lesssim \sqrt{p} g(t)^{1-\frac{1}{p}}
$$

Similarly, we have

$$
\begin{aligned}
|\mathrm{II}|=\int_{\mathbb{R}} w\left(P\left(D_{x}\right) w\right)(u+v) d x & \leq\left\|w^{1-\frac{2}{p}} P\left(D_{x}\right) w\right\|_{L^{\frac{p}{p-1}}}\left\|w^{\frac{2}{p}}(u+v)\right\|_{L^{p}} \\
& \lesssim\left\|w^{1-\frac{2}{p}}\right\|_{L^{\frac{2 p}{p-2}}}\left\|P\left(D_{x}\right) w\right\|_{L^{2}}\left(\|u\|_{L^{4}}^{\frac{2}{p}}+\|v\|_{L^{4}}^{\frac{2}{p}}\right)\|u+v\|_{L^{2 p}} \\
& \lesssim\|w\|_{L^{2}}^{1-\frac{2}{p}}\|w\|_{L^{2}}\left(\|u\|_{L^{2 p}}+\|v\|_{L^{2 p}}\right) \\
& \lesssim g(t)^{1-\frac{1}{p}}\left(\|u\|_{L^{2 p}}+\|v\|_{L^{2 p}} \lesssim \sqrt{p} g(t)^{1-\frac{1}{p}}\|u\|_{H^{\frac{1}{2}}} \lesssim \sqrt{p} g(t)^{1-\frac{1}{p}} .\right.
\end{aligned}
$$

We can write thanks to the fractional Leibniz rule [14].

Lemma 1.3. We have for $\frac{1}{p}=\frac{1}{q}+\frac{1}{r}$

$$
\|P(D)(u v)-v P(D)(u)-u P(D)(v)\|_{L^{p}} \lesssim\|u\|_{L^{q}}\|v\|_{L^{r}} .
$$


We deduce

$$
|\mathrm{III}|=\int_{\mathbb{R}} w R d x \lesssim\|w\|_{L^{2}}\|u+v\|_{L^{q}}\|w\|_{L^{r}} \lesssim\|w\|_{L^{2}}\left(\|u\|_{L^{q}}+\|v\|_{L^{q}}\right)\|w\|_{L^{2}}^{1-\frac{2}{p}}\|w\|_{L^{p}}^{\frac{2}{p}}
$$

where

$$
\frac{1}{r}=\frac{1}{2}-\frac{1}{q} \text { or } \frac{1}{r}=\frac{1}{2}-\frac{1}{p}\left(1-\frac{2}{p}\right) .
$$

Thus

$$
|\mathrm{III}| \lesssim \sqrt{q}\|w\|_{L^{2}}^{2-\frac{2}{p}}\|w\|_{L^{p}}^{\frac{2}{p}}\|u\|_{H^{\frac{1}{2}}} \lesssim \sqrt{q} g(t)^{1-\frac{1}{p}}\left(\|u\|_{L^{p}}^{\frac{2}{p}}+\|v\|_{L^{p}}^{\frac{2}{p}}\right) \lesssim p^{\frac{1}{p}} \sqrt{q} g(t)^{1-\frac{1}{p}}\|u\|_{H^{\frac{1}{2}}}^{\frac{2}{p}} \lesssim p^{\frac{1}{p}} \sqrt{q} g(t)^{1-\frac{1}{p}}
$$

Taking $p>2$ large enough so that

$$
|\mathrm{III}| \lesssim p^{\frac{1}{p}} \sqrt{\frac{p}{1-\frac{2}{p}}} g(t)^{1-\frac{1}{p}} \lesssim \sqrt{p} g(t)^{1-\frac{1}{p}}
$$

implies

$$
\left|g^{\prime}(t)\right| \leq C \sqrt{p} g(t)^{1-\frac{1}{p}}, \quad \forall 2<p<\infty,
$$

with $C=C\left(\left\|u_{0}\right\|_{H^{\frac{1}{2}}}, T\right)$ independent of $p$. Then

$$
g(t)^{\frac{1}{p}} \leq C \frac{t}{\sqrt{p}}
$$

or

$$
g(t) \leq C \frac{t^{p}}{p^{\frac{p}{2}}} \rightarrow 0, \quad \text { as } p \rightarrow \infty .
$$

Finally, $g(t) \equiv 0$, and $u=v$.

\section{THE WEAK DISPERSIVE EQUATION}

Let us come back to the initial value problem, for $0<\alpha<1$,

$$
\left\{\begin{array}{l}
u_{t}+u_{x}+u u_{x}+D_{x}^{\alpha} \partial_{t} u=0, \\
u(x, 0)=u_{0}(x)
\end{array}\right.
$$

The existence is proved in [16] using energy estimates.

Theorem 2.1. Let $0<\alpha<1, r>\max \left(1, \frac{3}{2}-\alpha\right)$ and $u_{0} \in H^{r}(\mathbb{R})$. Then the Cauchy problem has at least one solution in $H^{r}(\mathbb{R})$.

We briefly remind the proof in order to highlight the loss of uniqueness (see [16] for details).

Proof. For $r=s+\frac{\alpha}{2}$, we define $J^{s}=(I-\Delta)^{\frac{s}{2}}$ by its Fourier transform

$$
\widehat{J^{s} u}(\xi):=\left(\widehat{I-\Delta)^{\frac{s}{2}}} u(\xi)=\left(1+\xi^{2}\right)^{\frac{s}{2}} \widehat{u}(\xi)\right.
$$

Applying the operator $J^{s}$ to (3), multiplying by $J^{s} u$ and integrating by part over space, we obtain

$$
\begin{aligned}
\frac{1}{2} \frac{d}{d t} \int_{\mathbb{R}}\left|J^{s} u\right|^{2}+\left|J^{s} D_{x}^{\frac{\alpha}{2}} u\right|^{2} d x & =\int_{\mathbb{R}}-J^{s} u J^{s}\left(u u_{x}\right) d x \\
& =\int_{\mathbb{R}}\left|J^{s} u\right|^{2} u_{x}+u J^{s} u J^{s} \partial_{x} u+R J^{s} u d x=\frac{1}{2} \int_{\mathbb{R}}\left|J^{s} u\right|^{2} u_{x}+\int_{\mathbb{R}} R J^{s} u d x,
\end{aligned}
$$


where, thanks to Leibniz's rule,

$$
J^{s}\left(u u_{x}\right)=u J^{s} u_{x}+u_{x} J^{s} u+R
$$

with, for all $0<\epsilon<s$,

The Hölder inequality provides

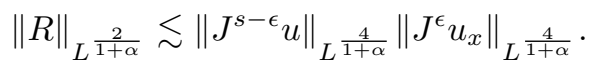

$$
\int_{\mathbb{R}}\left|\left(J^{s} u\right)^{2} u_{x}\right| d x \leq\left\|u_{x}\right\|_{L^{\frac{2}{1+\alpha}}}\left\|J^{s} u\right\|_{L^{\frac{2}{1-\alpha}}}^{2}
$$

and

Using Sobolev's embedding

$$
\int_{\mathbb{R}}\left|R J^{s} u\right| d x \leq\|R\|_{L^{\frac{2}{1+\alpha}}}\left\|J^{s} u\right\|_{L^{\frac{2}{1-\alpha}}} .
$$

$$
H^{\frac{\alpha}{2}}(\mathbb{R}) \hookrightarrow L^{\frac{2}{1-\alpha}}, H^{\frac{\alpha}{2}+\epsilon} \hookrightarrow L^{\frac{4}{1+\alpha}}, H^{s+\frac{\alpha}{2}-\epsilon-1} \hookrightarrow L^{\frac{4}{1+\alpha}},
$$

we finally find

and we conclude with the Gronwall lemma.

$$
\frac{d}{d t}\|u\|_{H^{s+\frac{\alpha}{2}}}^{2} \lesssim\|u\|_{H^{s+\frac{\alpha}{2}}}^{2}
$$

Let us try to prove the uniqueness. Let $u$ and $v$ be two solutions of (3) and denote $w=u-v$. We have

$$
w_{t}+\partial_{x}\left(1+D_{x}^{\alpha}\right)^{-1}\left(w+\frac{1}{2} w(u+v)\right)=0 .
$$

Using the fractional Leibniz rule and integrating by parts, it gets

$$
\begin{aligned}
\frac{1}{2} \int_{\mathbb{R}}\left|J^{s} w\right|^{2}+\left|J^{s+\frac{\alpha}{2}} w\right|^{2} d x \leq & \int_{\mathbb{R}}\left|J^{s}(u+v) w_{x} J^{s} w\right| d x+\frac{1}{2} \int_{\mathbb{R}}\left|(u+v)_{x}\right|\left|J^{s} w\right|^{2} d x \\
& +\int_{\mathbb{R}}\left|R J^{s} w\right| d x+\int_{\mathbb{R}}\left|J^{s}(u+v)_{x} w J^{s} w\right| d x .
\end{aligned}
$$

Here, for all $0<\epsilon<s$ and $\frac{1}{p}+\frac{1}{q}=\frac{1}{2}$,

$$
\|R\|_{L^{2}} \leq\left\|J^{s-\epsilon}(u+v)\right\|_{L^{p}}\left\|J^{\epsilon} w\right\|_{L^{q}}
$$

Even if the three first terms are bounded by $\|u+v\|_{H^{s+\frac{\alpha}{2}}}\|w\|_{H^{s+\frac{\alpha}{2}}}^{2}$, the last one can not be uniformly controlled by the $H^{s+\frac{\alpha}{2}}-$ norm when $0<\alpha<1$.

To avoid this difficulty, we propose the following regularization [11]

$$
\left\{\begin{array}{l}
u_{t}^{\varepsilon}+u_{x}^{\varepsilon}+u^{\varepsilon} u_{x}^{\varepsilon}+D_{x}^{\alpha} u_{t}^{\varepsilon}-\varepsilon u_{x x}^{\varepsilon}=0 \\
u^{\varepsilon}(0, x)=u_{0}^{\varepsilon}(x)
\end{array}\right.
$$

Lemma 2.2. Let $0<\alpha<1$ and $r \geq 0$. We have

$$
\left\|\partial_{x}\left(1+D_{x}^{\alpha}\right)^{-1} S_{t}(u v)\right\|_{H^{r}} \leq C(\varepsilon, t)\|u\|_{H^{r}}\|v\|_{H^{r}},
$$

where

$$
S_{t} u=\mathcal{F}^{-1}\left(\mathrm{e}^{-\frac{i \xi+\varepsilon \xi^{2}}{1+|\xi|^{\alpha}} t}\right) * u(x), \text { and } C(\varepsilon, t)=C(\varepsilon t)^{-\frac{7}{4}-\alpha}
$$


Proof. The idea of the proof is introduced in $[4,13]$. By duality, it is enough to show that for all function $w \in \mathcal{S}(\mathbb{R})$,

$$
\int_{\mathbb{R}} \partial_{x}\left(1+D_{x}^{\alpha}\right)^{-1} S_{t}(u(x) v(x)) \bar{w}(x) d x \leq C(t)\|u\|_{H^{r}}\|v\|_{H^{r}}\|w\|_{H^{-r}} .
$$

The Plancherel identity provides

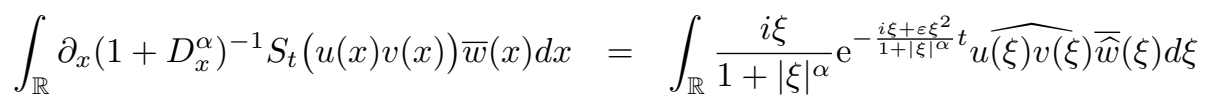

$$
\begin{aligned}
& =\int_{\mathbb{R}} \frac{i \xi}{1+|\xi|^{\alpha}} \mathrm{e}^{-\frac{i \xi+\varepsilon \xi^{2}}{1+|\xi| \alpha} \widehat{u} * \widehat{v}(\xi) \widehat{\widehat{w}}(\xi) d \xi} \\
& =\int_{\mathbb{R}} \int_{\mathbb{R}} \frac{i \xi}{1+|\xi|^{\alpha}} \mathrm{e}^{-\frac{i \xi+\varepsilon \xi^{2}}{1+|\xi|^{\alpha}}} \widehat{u}(\xi-\eta) \widehat{v}(\eta) d \eta \overline{\widehat{w}}(\xi) d \xi .
\end{aligned}
$$

Let us denote $\langle\xi\rangle=\left(1+\xi^{2}\right)^{\frac{1}{2}}, \widehat{U}=\langle\xi\rangle^{r} \widehat{u}, \widehat{V}=\langle\xi\rangle^{r} \widehat{v}, \widehat{W}=\langle\xi\rangle^{-r} \widehat{w}$, it is necessary to prove

$$
\left|\int_{\mathbb{R}} \int_{\mathbb{R}} e^{-\frac{i \xi+\varepsilon \xi^{2}}{1+|\xi|^{\alpha}} t} \frac{|\xi|\langle\xi\rangle^{r}}{\langle\xi\rangle^{\alpha}\langle\xi-\eta\rangle^{r}\langle\eta\rangle^{r}} \widehat{U}(\xi-\eta) \widehat{V}(\eta) \widehat{\widehat{W}}(\xi) d \xi d \eta\right| \leq C(\varepsilon, t)\|u\|_{H^{r}}\|v\|_{H^{r}}\|w\|_{H^{-r}} .
$$

The triangle inequality $\langle\xi\rangle^{r} \lesssim\langle\xi-\eta\rangle^{r}\langle\eta\rangle^{r}$ implies

$$
\begin{aligned}
&\left|\int_{\mathbb{R}} \int_{\mathbb{R}} e^{-\frac{i \xi+\varepsilon \xi^{2}}{1+|\xi|^{\alpha}} t} \frac{|\xi|\langle\xi\rangle^{r}}{\langle\xi\rangle^{\alpha}\langle\xi-\eta\rangle^{r}\langle\eta\rangle^{r}} \widehat{U}(\xi-\eta) \widehat{V}(\eta) \widehat{\widehat{W}}(\xi) d \xi d \eta\right| \\
& \quad \lesssim \int_{\mathbb{R}} \int_{\mathbb{R}}\langle\xi\rangle^{1-\alpha} e^{-\frac{\varepsilon \xi^{2}}{1+|\xi| \alpha} t}|\widehat{U}(\xi-\eta) \widehat{V}(\eta) \widehat{\widehat{W}}(\xi)| d \xi d \eta \\
& \quad \lesssim\|W\|_{L_{\xi}^{2}}\left\|\langle\xi\rangle^{1-\alpha} e^{-\frac{\varepsilon \xi^{2}}{1+|\xi|^{\alpha}} t} \int_{\mathbb{R}} \widehat{U}(\xi-\eta) \widehat{V}(\eta) d \eta\right\|_{L_{\xi}^{2}} \\
& \lesssim\|W\|_{L_{\xi}^{2}}\left\|\langle\xi\rangle^{1-\alpha} e^{-\frac{\varepsilon \xi^{2}}{1+|\xi|^{\alpha}} t}\right\|_{L_{\xi}^{2}}\left\|\int_{\mathbb{R}} \widehat{U}(\xi-\eta) \widehat{V}(\eta) d \eta\right\|_{L_{\xi}^{\infty}} \\
& \lesssim\|W\|_{L_{\xi}^{2}}\|U\|_{L_{\xi}^{2}}\|V\|_{L_{\xi}^{2}}\left\|\langle\xi\rangle^{1-\alpha} e^{-\frac{\varepsilon \xi^{2}}{1+|\xi| \alpha} t}\right\|_{L_{\xi}^{2}} \\
& \lesssim\|w\|_{H^{-r}}\|u\|_{H^{r}}\|v\|_{H^{r}}\left\|\langle\xi\rangle^{1-\alpha} e^{-\frac{\varepsilon \xi^{2}}{1+|\xi| \alpha} t}\right\|_{L_{\xi}^{2}} .
\end{aligned}
$$

Since for $x \in \mathbb{R}, \theta \in \mathbb{R}$, we have $e^{-x} \leq \frac{1}{x^{\theta}}$, we deduce

$$
\left\|\langle\xi\rangle^{1-\alpha} e^{-\frac{\varepsilon \xi^{2}}{1+|\xi|^{\alpha}} t}\right\|_{L_{\xi}^{2}} \leq\left\|\langle\xi\rangle^{1-\alpha} \frac{1}{\left(\frac{\varepsilon \xi^{2} t}{1+|\xi|^{\alpha}}\right)^{\theta}}\right\|_{L_{\xi}^{2}} \leq \frac{1}{(\varepsilon t)^{\theta}}\left\|\frac{1}{\langle\xi\rangle^{(2-\alpha) \theta-(1-\alpha)}}\right\|_{L_{\xi}^{2}} \lesssim \frac{1}{(\varepsilon t)^{\theta}},
$$

if $(2-\alpha) \theta-(1-\alpha)>1 / 2$, i.e. $\theta>\frac{\frac{3}{2}-\alpha}{2-\alpha}$.

Lemma 2.3. Let $0<\alpha<1, r \geq 0$ and $u_{0}^{\varepsilon} \in H^{r}(\mathbb{R})$, then there exist a time $T_{\varepsilon}=T(\varepsilon)>0$ and a unique solution $u^{\varepsilon} \in \mathcal{C}\left(\left[0, T_{\varepsilon}\right] ; H^{r}(\mathbb{R})\right)$.

Proof. Thanks to the Duhamel formula, $u^{\varepsilon}$ is solution of (4) if and only if $u^{\varepsilon}$ is the fixed point of $\Phi^{\varepsilon}$ defined as

$$
\Phi^{\varepsilon} u^{\varepsilon}(t):=S_{t} u_{0}^{\varepsilon}-\frac{1}{2} \int_{0}^{t} S_{t-\tau}\left(\left(1+D_{x}^{\alpha}\right)^{-1} \partial_{x} u^{\varepsilon}(\tau)^{2}\right) d \tau
$$


where $S_{t} u=\mathcal{F}^{-1}\left(e^{-\frac{i \xi+\varepsilon \xi^{2}}{1+|\xi| \alpha}}\right) * u(x)$. Let $\bar{B}_{T}$ be the closed ball

$$
\bar{B}_{T}:=\left\{u \in \mathcal{C}\left([0, T] ; H^{r}(\mathbb{R})\right),\|u\|_{L^{\infty}\left([0, T], H^{r}(\mathbb{R})\right)} \leq 2\left\|u_{0}\right\|_{H^{r}}\right\} .
$$

We prove that $\Phi^{\varepsilon}\left(\bar{B}_{T}\right) \subseteq \bar{B}_{T}$ and $\Phi^{\varepsilon}$ is a contraction mapping on $\bar{B}_{T}$. Let $u^{\varepsilon} \in \bar{B}_{T}$, we have

$$
\begin{aligned}
\left\|\Phi^{\varepsilon} u^{\varepsilon}(t)\right\|_{H^{r}(\mathbb{R})} & =\left\|S_{t} u_{0}-\frac{1}{2} \int_{0}^{t} S_{t-\tau}\left(1+D_{x}^{\alpha}\right)^{-1} \partial_{x} u^{\varepsilon}(\tau)^{2} d \tau\right\|_{H^{r}} \\
& \leq\left\|u_{0}^{\varepsilon}\right\|_{H^{r}}+\int_{0}^{t}\left\|S_{t-\tau}\left(1+D_{x}^{\alpha}\right)^{-1} \partial_{x} u^{\varepsilon}(\tau)^{2}\right\|_{H^{r}} d \tau .
\end{aligned}
$$

Lemma 2.2 gives

$$
\left\|S_{t-\tau}\left(1+D_{x}^{\alpha}\right)^{-1} \partial_{x} u^{\varepsilon}(\tau)^{2}\right\|_{H^{r}} \leq C(\varepsilon(t-\tau))^{-\frac{7}{2-\alpha}}\left\|u^{\varepsilon}(\tau)\right\|_{H^{r}}^{2} .
$$

Note that $0<\frac{\frac{7}{4}-\alpha}{2-\alpha}<1$, and

$$
\left\|\Phi^{\varepsilon} u^{\varepsilon}(t)\right\|_{H^{r}} \leq\left\|u_{0}^{\varepsilon}\right\|_{H^{r}}+C_{\varepsilon, r} T\left\|u^{\varepsilon}\right\|_{L^{\infty}\left([0, T], H^{r}\right)}^{2} .
$$

Thus, choosing $T<\frac{1}{C_{\varepsilon, r}\left\|u_{0}^{\varepsilon}\right\|_{H^{r}}}$ implies

$$
\left\|\Phi^{\varepsilon} u^{\varepsilon}(t)\right\|_{L^{\infty}\left([0, T], H^{r}\right)} \leq 2\left\|u_{0}^{\varepsilon}\right\|_{H^{r}}
$$

Let $u_{1}^{\varepsilon}, u_{2}^{\varepsilon} \in \bar{B}_{T}$, one gets from Lemma 2.2

$$
\begin{aligned}
\left\|\Phi^{\varepsilon} u_{1}^{\varepsilon}(t)-\Phi^{\varepsilon} u_{2}^{\varepsilon}(t)\right\|_{H^{r}} & \leq \int_{0}^{t}\left\|S_{t-\tau}\left(1+D_{x}^{\alpha}\right)^{-1} \partial_{x}\left(\left(u_{1}^{\varepsilon}\right)^{2}-\left(u_{2}^{\varepsilon}\right)^{2}\right)\right\|_{H^{r}} d \tau \\
& \leq \int_{0}^{t} C(\varepsilon(t-\tau))^{-\frac{7}{2}-\alpha}\left\|u_{1}^{\varepsilon}+u_{2}^{\varepsilon}\right\|_{H^{r}}\left\|u_{1}^{\varepsilon}-u_{2}^{\varepsilon}\right\|_{H^{r}} d \tau \\
& \leq C_{\varepsilon, r} T\left\|u_{0}^{\varepsilon}\right\|_{H^{r}}\left\|u_{1}^{\varepsilon}-u_{2}^{\varepsilon}\right\|_{L^{\infty}\left([0, T] ; H^{r}\right)},
\end{aligned}
$$

and $\Phi^{\varepsilon}$ is a contraction mapping on $\bar{B}_{T}$ as soon as $T<\frac{1}{C_{\varepsilon, r}\left\|u_{0}^{\varepsilon}\right\|_{H^{r}}}$. Finally, there exists a unique fixed point $u^{\varepsilon}:=\Phi^{\varepsilon}\left(u^{\varepsilon}\right)$ in $\bar{B}_{T}$ for $T<\frac{1}{C_{\varepsilon, s}\left\|u_{0}^{\varepsilon}\right\|_{H^{r}}}$.

To obtain the continuity with respect to the initial data, for $u_{0}^{\varepsilon}$ and $v_{0}^{\varepsilon}$ in $H^{r}(\mathbb{R})$ with $\left\|u_{0}^{\varepsilon}\right\|_{H^{r}} \leq M$, $\left\|v_{0}^{\varepsilon}\right\|_{H^{r}} \leq M$, we consider $u^{\varepsilon}, v^{\varepsilon}$ the respective solution. Let $0 \leq t \leq T=\frac{1}{C_{\varepsilon, s} M}$, we find similarly

$$
\begin{aligned}
\left\|u^{\varepsilon}(t)-v^{\varepsilon}(t)\right\|_{H^{r}} & \leq\left\|S_{t}\left(u_{0}^{\varepsilon}-v_{0}^{\varepsilon}\right)\right\|_{H^{r}}+\int_{0}^{t}\left\|S_{t-\tau}\left(1+D_{x}^{\alpha}\right)^{-1} \partial_{x}\left(\left(u^{\varepsilon}\right)^{2}-\left(v^{\varepsilon}\right)^{2}\right)\right\|_{H^{r}} d \tau \\
& \leq\left\|u_{0}^{\varepsilon}-v_{0}^{\varepsilon}\right\|_{H^{r}}+C_{\varepsilon, r} T M\left\|u^{\varepsilon}-v^{\varepsilon}\right\|_{L^{\infty}\left([0, T] ; H^{r}\right)},
\end{aligned}
$$

or in other words, since $1-C_{\varepsilon, r} T M>0$,

$$
\left\|u^{\varepsilon}(t)-v^{\varepsilon}(t)\right\|_{H^{r}} \leq \frac{1}{1-C_{\varepsilon, r} T M}\left\|u_{0}^{\varepsilon}-v_{0}^{\varepsilon}\right\|_{H^{r}} .
$$

Lemma 2.4. Let $0<\alpha<1, r \geq 2-\frac{\alpha}{2}$ and $u_{0}^{\varepsilon} \in H^{r}(\mathbb{R})$, then $T_{\varepsilon}$ can be chosen independent of $\varepsilon$. 
Proof. Let $r=s+\frac{\alpha}{2}$. Applying $J^{s}$ to (4), multiplying by $J^{s} u^{\varepsilon}$ and integrating by parts over space, it comes

$$
\frac{d}{d t}\left(\int_{\mathbb{R}}\left(J^{s} u^{\varepsilon}\right)^{2}+\left(J^{s} D_{x}^{\frac{\alpha}{2}} u^{\varepsilon}\right)^{2} d x\right)+2 \varepsilon \int_{\mathbb{R}}\left(J^{s} u_{x}^{\varepsilon}\right)^{2} d x=\int_{\mathbb{R}}-J^{s} u^{\varepsilon} J^{s}\left(u^{\varepsilon} u_{x}^{\varepsilon}\right) d x .
$$

We obtain from Kato-Ponce commutator estimates [12]

$$
\int_{\mathbb{R}} J^{s} u^{\varepsilon} J^{s}\left(u^{\varepsilon} u_{x}^{\varepsilon}\right) d x=\frac{1}{2} \int_{\mathbb{R}} u_{x}^{\varepsilon}\left(J^{s} u^{\varepsilon}\right)^{2} d x+\int_{\mathbb{R}} R J^{s} u^{\varepsilon} d x \leq C\left\|u^{\varepsilon}\right\|_{H^{s+\frac{\alpha}{2}}}^{3}
$$

It follows that

$$
\frac{d}{d t}\left\|u^{\varepsilon}\right\|_{H^{s+\frac{\alpha}{2}}}^{2} \leq C\left\|u^{\varepsilon}\right\|_{H^{s+\frac{\alpha}{2}}}^{3}
$$

or in other words $\left\|u^{\varepsilon}\right\|_{H^{s+\frac{\alpha}{2}}}^{2} \leq y(t)$ where $y(t)$ satisfies the ordinary differential equation

$$
\left\{\begin{array}{l}
y^{\prime}(t)=C y(t)^{\frac{3}{2}} \\
y(0)=\left\|u_{0}^{\varepsilon}\right\|_{H^{s+\frac{\alpha}{2}}(\mathbb{R})}^{2},
\end{array}\right.
$$

which solution is given by

$$
y(t)=\frac{y(0)}{\left(1-C y(0)^{1 / 2} t\right)^{2}}=\frac{\left\|u_{0}^{\varepsilon}\right\|_{H^{s+\frac{\alpha}{2}}}^{2}}{\left(1-C\left\|u_{0}^{\varepsilon}\right\|_{H^{s+\frac{\alpha}{2}}} t\right)^{2}},
$$

and $u^{\varepsilon}$ can be extended until $T=\frac{1}{2 C\left\|u_{0}^{\varepsilon}\right\|_{H^{s+\frac{\alpha}{2}}}}$.

Theorem 2.5. Let $0<\alpha<1, r \geq 2-\frac{\alpha}{2}$ and $u_{0} \in H^{r}(\mathbb{R})$. Then there exist a time $T>0$ and a unique vanishing viscosity solution $u \in \mathcal{C}\left([0, T] ; H^{r}(\mathbb{R})\right)$ of the initial value problem (3). In other words, the solution $u^{\varepsilon}$ of the initial value problem (4) converges, when $\varepsilon \rightarrow 0$, uniformly to u solution of $(3)$ in $\mathcal{C}\left([0, T] ; H^{r}(\mathbb{R})\right)$.

Proof. We prove that $\left(u^{\varepsilon}\right)_{\varepsilon \geq 0}$ is a Cauchy sequence in the Sobolev space $H^{r}(\mathbb{R})$. Let $u^{\varepsilon}$ and $v^{\delta}$ be two solutions of (4). The difference $w=u^{\varepsilon}-v^{\delta}$ satisfies

$$
w_{t}+w_{x}+D_{x}^{\alpha} \partial_{t} w-\delta w_{x x}+\left(u^{\varepsilon} w-\frac{w^{2}}{2}\right)_{x}=(\varepsilon-\delta) u_{x x}^{\varepsilon}
$$

One multiplies (5) by $w$ and integrates over space to obtain

$$
\frac{d}{d t} \int_{-\infty}^{+\infty} w^{2}+\left(D_{x}^{\frac{\alpha}{2}} w\right)^{2} d x=\int_{-\infty}^{+\infty}-2 \delta w_{x}^{2}-u_{x}^{\varepsilon} w^{2}+2(\varepsilon-\delta) u_{x x}^{\varepsilon} w d x \leq \int_{-\infty}^{+\infty}-u_{x}^{\varepsilon} w^{2}+2(\varepsilon-\delta) u_{x x}^{\varepsilon} w d x
$$

and Sobolev's inequality provide

$$
\frac{d}{d t} \int_{-\infty}^{+\infty} w^{2}+\left(D_{x}^{\frac{\alpha}{2}} w\right)^{2} d x \leq C\|u\|_{H^{2-\frac{\alpha}{2}}}\|w\|_{H^{\frac{\alpha}{2}}}^{2}+C(\varepsilon-\delta)\|u\|_{H^{2-\frac{\alpha}{2}}}\|w\|_{H^{\frac{\alpha}{2}}} .
$$

Finally

$$
\frac{d}{d t}\|w\|_{H^{\frac{\alpha}{2}}}^{2} \leq C\|w\|_{H^{\frac{\alpha}{2}}}^{2}+C(\varepsilon-\delta)\|w\|_{H^{\frac{\alpha}{2}}}
$$

and the Gronwall Lemma offers a limit of $\left(u^{\varepsilon}\right)_{\varepsilon \geq 0}$ in $H^{\frac{\alpha}{2}}(\mathbb{R})$.

From the preceding lemma, the map $t \in[0, T] \rightarrow u^{\varepsilon}(t)$ is continuous and uniformly bounded. In particular, the sequence $\left(u^{\varepsilon}(t)\right)_{\varepsilon \geq 0}$ is weakly convergent in $H^{s}(\mathbb{R}), s \geq \frac{\alpha}{2}$ to $u(t)$ a weakly continuous and uniformly bounded function. We deduce

$$
t \in[0, T] \rightarrow\left(1+D_{x}^{\alpha}\right)^{-1}\left(\partial_{x} u+u \partial_{x} u\right) \in H^{s+1-\alpha}(\mathbb{R})
$$


is weakly continuous and

$$
u(t)=u_{0}-\int_{0}^{t}\left(1+D_{x}^{\alpha}\right)^{-1}\left(\partial_{x} u+u \partial_{x} u\right) \text { in } H^{s+1-\alpha}(\mathbb{R})
$$

is unique. Indeed, let $v \in H^{s+1-\alpha}(\mathbb{R})$ defined by

$$
v(t)=v_{0}-\int_{0}^{t}\left(1+D_{x}^{\alpha}\right)^{-1}\left(\partial_{x} v+v \partial_{x} v\right)
$$

we obtain with similar computations

$$
\frac{d}{d t}\|u-v\|_{H^{\frac{\alpha}{2}}}^{2} \leq C\|u-v\|_{H^{\frac{\alpha}{2}}}^{2}
$$

and the Gronwall lemma allows to conclude.

\section{REFERENCES}

[1] J.L. Bona, And R. Smith, The initial-value problem for the Korteweg-de Vries equation, Philos. Trans. Roy. Soc. London Ser. A 278, 1287 (1975) 555-601.

[2] T.B. Benjamin, J.L. Bona, And J.J Mahony, Model equations for long waves in nonlinear dispersive systems, Philos. Trans. Roy. Soc. London Ser. A 272, 1220 (1972) 47-78.

[3] J.L. Bona, And J. C. SAut, Dispersive blow-up II. Schrödinger-type equations, optical and oceanic rogue waves, Chinese Ann. Math. Ser. B 31, 6 (2010) 793-818.

[4] J.L. Bona, And N. Tzvetkov, Sharp well-posedness results for the BBM equation, Discrete Contin. Dyn. Syst 23, 7 (2009) 1241-1252.

[5] H. BrÉzis, And T. Gallouët, Nonlinear Schrödinger evolution equations, Nonlinear Anal. 4 (1980) 677-681.

[6] N. Burq, And F. Planchon, On well-posedness for the Benjamin-Ono equation, Mathematische Annalen 340, 3 (2008) 497-542.

[7] J. Colliander, M. Keel, G. Staffilani, H. Takaoka, and T. TaO, Sharp global well-posedness for KdV and modified KdV on $\mathbb{R}$ and $\mathbb{T}$, Journal American Math. Soc. 16, 3 (2003) 705-749.

[8] A. Friedman, Partial differential equations of parabolic type, Courier Corporation, 2013.

[9] P. Gérard, and S. Grellier, The cubic Szegö equation, Annales École Normale Sup. 43, 5 (2010) 761-810.

[10] A. Ionescu, And C. Kenig, Global well-posedness of the Benjamin-Ono equation in low-regularity spaces, Journal American Math. Soc. 20,3 (2007) 753-798.

[11] R.J. Iório JR., KdV, BO and friends in weighted Sobolev spaces, Functional-Analytic Methods for Partial Differential equations, Lecture Notes in Math. 1450, Springer-Verlag, Berlin, (1990) 104-121.

[12] T. Kato, And G. Ponce, Commutator estimates and the Euler and Navier-Stokes equations, Commun. Pure Appl. Math. 41,7 (1988) 891-907.

[13] C.E. Kenig, G. Ponce, And L. Vega, A bilinear estimate with applications to the KdV equation, J. American Math. Soc. 9,2 (1996) 573-603.

[14] C.E. Kenig, G. Ponce, And L. Vega, Well-posedness and scattering results for the generalized Korteweg-de Vries equation via the contraction principle, Commun. Pure Appl. Math. 46, 4 (1993) 527-620.

[15] C. Klein, And J.C. Saut, A numerical approach to blow-up issues for dispersive perturbations of Burgers' equation, Physica D 295 (2015) 46-65.

[16] F. Linares, D. Pilod, and J.C. Saut, Dispersive perturbations of Burgers and hyperbolic equations I: local theory, SIAM Journal Math. Ana. 46, 2 (2014) 1505-1537.

[17] Y. MAmmeri, On the decay in time of solutions of some generalized regularized long waves equations, CPAA Comm. Pure Appl. Ana. 7, 3, (2008), 513-532.

[18] Y. MammerI, Long time bounds for the periodic Benjamin-Ono-BBM equation, Nonlinear Analysis TMA 71, 10 (2009) 5010-5021.

[19] E. Stein, Harmonic Analysis: Real-variable Methods, Orthogonality, and Oscillatory Integrals, Princeton University Press, (1993).

[20] T. TAO, Global well-posedness of the Benjamin-Ono equation in $H^{1}(R)$, Journal Hyperbolic Diff. Eq. 1 (2004) $27-49$.

[21] V.I. Yudovich, Non-stationary flows of an ideal incompressible fluid, Zhurnal Vychislitel'noi Matematikii Matematicheskoi Fiziki, 3, 6 (1963) 1032-1066. 